\title{
Noether's Inequality for Non-complete Algebraic Surfaces of General Type
}

By

\author{
Shuichiro TsunODA* and De-Qi ZHANG*
}

\begin{abstract}
Let $V$ be a nonsingular projective surface. M. Noether proved that $\operatorname{dim} H^{0}\left(V, K_{V}\right)$ $\leqq 1 / 2\left(K_{V}^{2}\right)+2$, where $K_{V}$ is the canonical divisor of $V$, provided $V$ is a minimal surface of general type. Let $D$ be a reduced, effective divisor on $V$ with only simple normal crossings. An open surface $V-D$ is said to be of general type if the Kodaira dimension $\kappa\left(V, K_{v}+D\right)=2$. In this case, $K_{V}+D$ has the Zariski decomposition and we denote by $P$, which is a $\boldsymbol{Q}$-divisor, the numerically effective part of the decomposition. We have $\left(P^{2}\right)=\left(c_{1}(V)^{2}\right)$ if $D=0$ and if $V$ is a minimal surface of general type. In the present article, we shall verify that $\operatorname{dim} H^{0}\left(V, K_{V}+D\right) \leqq 9 / 8\left(P^{2}\right)+2$ and several other inequalities. Such pairs $(V, D)$ that the above inequality becomes an equality are precisely described. The case that $D$ is semi-stable has been treated by Sakai [Math. Ann. 254, 89-120 (1980)].
\end{abstract}

\section{Introduction}

Let $k$ be an algebraically closed field of characteristic zero, which we fix as a ground field throughout this article. Let $V$ be a nonsingular projective surface defined over $k$. If $V$ is a minimal surface of general type, we have the following inequality due to $\mathrm{M}$. Noether:

$$
p_{g}(V) \leqq \frac{1}{2} c_{1}(V)^{2}+2 \text {. }
$$

We intend, in the present article, to extend this inequality to a logarithmic surface of general type, which is to be defined below.

Let $V$ be a nonsingular projective surface defined over $k$ and let $D$ be a reduced effective divisor on $V$ with only simple normal crossings. Denote by $K_{V}$ the canonical divisor of $V$. Let $D=\Sigma D_{i}$ be the decomposition into irreducible components. We call a pair $\left(I^{r}, D\right)$ a logarithmic surface (log surface, for short). It is called minimal if $K_{V}+D$ has a decomposition into a sum of $\boldsymbol{Q}$ divisors :

$$
K_{V}+D=\left(K_{V}+\sum \alpha_{i} D_{\imath}\right)+\Sigma\left(1-\alpha_{i}\right) D_{\imath}, \quad \text { where }
$$

Communicated by K. Saito, December 25, 1990.

1991 Mathematics Subject Classification : 14J29.

* Department of Mathematics, Osaka University, Toyonaka, Osaka 560, Japan. 
(1) $0 \leqq \alpha_{i} \leqq 1$ and $\alpha_{i} \in \boldsymbol{Q}$,

(2) $K_{V}+\sum \alpha_{i} D_{i}$ is numerically effective,

(3) the intersection matrix of $\Sigma\left(1-\alpha_{i}\right) D_{i}$ is negative definite, and

(4) if $\alpha_{\jmath} \neq 1$ then $\left(K_{V}+\sum \alpha_{i} D_{i}, D_{\jmath}\right)=0$ and $\left(D_{\jmath}^{2}\right) \leqq-2$.

This decomposition is unique and called the Zariski decomposition. We write $\sum \alpha_{i} D_{i}$ as $D^{*}$. Then the surface $(V, D)$ is a logarithmic surface of general type if $\left(K_{V}+D^{*}\right)^{2}>0$ or synonymously if the Kodaira dimension $\kappa\left(V, K_{V}+D\right)$ is equal to 2. The connected components of $\operatorname{Supp}\left(D-D^{*}\right)$ consist of twigs, rods or forks (see Miyanishi and Tsunoda $[4 ;$ p. 436] for the relevant definitions), which can be contracted to normal points with quotient singularities. We denote $\operatorname{dim} H^{0}\left(V, K_{V}+D\right)$ by $\bar{p}_{g}$ and $\left(K_{V}+D^{*}\right)^{2}$ by $\left(\tilde{c}_{1}^{2}\right)$.

Now we can state the following results.

Theorem 1.3. Let $(V, D)$ be a minimal $\log$ surface of general type such thal $\left|K_{V}+D\right|$ is composed with a pencil. Then we have:

$$
\bar{p}_{g} \leqq \frac{1}{2}\left\{\left(\bar{c}_{1}^{2}\right)+\sqrt{\left(\bar{c}_{1}^{2}\right)^{2}+8\left(\bar{c}_{1}^{2}\right)}\right\}+1 \leqq \frac{9}{8}\left(\bar{c}_{1}^{2}\right)+2 .
$$

We have also $\bar{p}_{g}<\left(\bar{c}_{1}^{2}\right)+3$.

Theorem 2.10. Let $(V, D)$ be a minimal log surface of general type such that $\bar{p}_{g} \geqq 3$ and $\left|K_{V}+D\right|$ is not composed with a pencil. Write $\left|K_{V}+D\right|=|C|+G$, where $|C|$ and $G$ are respectively the movable part and the fixed part of $\left|K_{V}+D\right|$. Then the following assertions hold, where $C$ is replaced by a general member of $|C|$ if necessary.

(1) We have $\bar{p}_{g} \leqq\left(\bar{c}_{1}^{2}\right)+2$. Suppose $\bar{p}_{g}=\left(\bar{c}_{1}^{2}\right)+2$. Then $C \sim K_{V}+D^{*}$ and $\Phi_{|C|}$ : $V \rightarrow \boldsymbol{P}^{N}\left(N:=\bar{p}_{g}-1\right)$ is a birational morphism onto a normal rational surface $\bar{W}$ of degree $N-1$. (See the precise description of $\bar{W}$ and $C$ in Lemmas 2.2 and 2.3.)

(2) Suppose $V$ is not a rational surface. Then $\bar{p}_{g} \leqq\left(\bar{c}_{1}^{2}\right)+1$. Moreover, if the equality holds then $C \sim K_{V}+D^{*}, \bar{p}_{g}=3,2 \leqq g(C)=p_{a}(C) \leqq 3,1+p_{g}(V) \leqq h^{1}(V, C)$ $+\chi\left(\mathcal{O}_{V}\right)=h^{0}\left(V,-D^{*}\right)+g(C)$ and $\Phi_{|C|}: V \rightarrow P^{2}$ is a morphism of degree two.

(3) Suppose $V$ is not a ruled surface. Then $\bar{p}_{g} \leqq 1 / 2\left(\bar{c}_{1}^{2}\right)+2$. Moreover, if the equality holds then $C \sim K_{V}+D^{*}, 1 / 2\left(C^{2}\right)+1 \leqq g(C)=p_{a}(C) \leqq\left(C^{2}\right)+1,1+p_{g}(V) \leqq$ $h^{1}(V, C)+\chi\left(\mathcal{O}_{V}\right)=h^{0}\left(V,-D^{*}\right)+g(C)+1-1 / 2\left(C^{2}\right)$ and either $\Phi_{|C|}: V \rightarrow P^{N}(N:=$ $\left.\bar{p}_{g}-1\right)$ is a birational morphism onto a surface of degree $2(N-1)$ or $\Phi_{|C|}: V \rightarrow$ $\boldsymbol{P}^{N}$ is a morphism of degree two onto a normal rational surface $\bar{W}$ of degree $N-1$.

Write $\left|K_{V}+D\right|=|C|+G$ as in Theorem 2.10. In $\S 1$ we consider the case where $|C|$ is composed with a pencil. After proving Theorem 1.3, we describe precisely in Propositions 1.6 and 1.7 such surfaces $(V, D)$ that the first inequality of Theorem 1.3 becomes an equality. In view of Example 1.8, the bounds for 
$\bar{p}_{g}$ in Theorem 1.3 are the best possible ones. In $\S 2$ we consider the case where $|C|$ is not composed with a pencil. At first, we prove several inequalities of the form $h^{0}(V, C) \leqq \alpha\left(C^{2}\right)+\beta$ with $\alpha, \beta \in \boldsymbol{Q}$ (cf. Theorem 2.8). We also consider the cases where these inequalities become equalities. Then Theorem 2.10 is a consequence of Theorem 2.8 and the fact $\left(K_{V}+D^{*}\right)^{2} \geqq\left(C^{2}\right)$ (cf. Lemma 2.9).

These theorems can be considered as one of Fujita's $\Delta$-genus type results (cf. [1]). In fact, there is a direct relation between Fujita's theorem and ours (see $\S 2$ ). On the other hand, Sakai [6] has treated the case where $D$ is semistable.

We shall use the following terminology and notation. Let $V$ be a nonsingular projective surface. If $E$ is a nonsingular rational curve on $V$ with $\left(E^{2}\right)=-n$, we shall call $E$ a $(-n)$-curve. A divisor $H$ on $V$ is called numerically effective (nef, for short) if the intersection number $(H, R) \geqq 0$ for every curve $R$. A nef divisor $H$ is called nef and big if the self intersection number $\left(H^{2}\right)>0$.

$K_{V}$ : Canonical divisor of $V$

$\kappa(V)$ : Kodaira dimension of $V$

$h^{\imath}(V, H):=\operatorname{dim} H^{\imath}(V, H)$

$p_{g}(V):=\operatorname{dim} H^{0}\left(V, K_{V}\right)$, the geometric genus of $V$

$\bar{p}_{g}:=\operatorname{dim} H^{0}\left(V, K_{V}+D\right)$

$q(V):=\operatorname{dim} H^{1}\left(V, \mathcal{O}_{V}\right)$, the irregularity of $V$

$p_{a}(A)$ : Arithmetic genus of an irreducible curve $A$

$g(A)$ : Geometric genus of an irreducible curve $A$

$|H|$ : Complete linear system defined by $H$

$\Phi_{|H|}$ : Rational map $V \rightarrow \boldsymbol{P}^{\operatorname{dim}|H|}$ defined by $|H|$

$f^{*}(H)$ : Total transform by a morphism $f$

$f^{\prime}(H)$ : Proper transform by a birational morphism $f$

$H_{1} \sim H_{2}: \quad H_{1}$ and $H_{2}$ are linearly equivalent divisors

$H_{1} \equiv H_{2}: \quad H_{1}$ and $H_{2}$ are numerically equivalent divisors

$\Sigma_{n}, M_{n}$ : A Hirzebruch surface $\Sigma_{n}$ of degree $n$ and a minimal section $M_{n}$ on $\Sigma_{n}$ satisfying $\left(M_{n}^{2}\right)=-n$.

The authors would like to express their gratitude to Professor M. Miyanishi for giving valuable advice during the preparation of the present article. The second author would also like to thank the Yukawa Foundation for partial financial support.

\section{$\S 1$. The Case where $\left|K_{V}+D\right|$ is Composed with a Pencil}

Let $(V, D)$ be a minimal surface of general type such that the movable part $|C|$ of $\left|K_{V}+D\right|$ is composed with a pencil. Namely, the rational map $\Phi_{1 C}$ : 
$V \rightarrow \boldsymbol{P}^{N}$ has a curve $B$ as the image. Let $\bar{B}$ be the normalization of $B$ in the function field $k(V)$ of $V$. So, we have a decomposition of $\Phi_{|C|}$ to a composite $\nu \cdot \varphi$, where $\varphi: V \rightarrow \bar{B}$ is a rational map whose general fibers are irreducible and $\nu: \bar{B} \rightarrow B$ is the normalization morphism. Let $d$ be the degree of the curve $B$ in $\boldsymbol{P}^{N}$ and let $e$ be the degree of the finite morphism $\nu$. Let $F$ be a general fiber of $\varphi$. Then it is well known that $d \geqq N$ and $C$ is numerically equivalent to $d e F$. Since $\bar{p}_{g}=N+1$, we have $n:=d e \geqq d \geqq \bar{p}_{g}-1$, i. e., $\bar{p}_{g} \leqq n+1$. Now $K_{V}+$ $D$ is written as $n F+G$ upto numerical equivalence.

As in the Introduction, write

$$
D=D^{*}+\sum_{i=1}^{t}\left(1-\alpha_{i}\right) D_{i}, \quad \text { where } \quad 0 \leqq \alpha_{\imath}<1 .
$$

Note that the intersection matrix $\left(\left(D_{\imath}, D_{j}\right)_{1 \leqq i, j \leqq t}\right.$ is negative definite. Define $\boldsymbol{Q}$-divisors $F^{*}$ and $G^{*}$ as follows :

$$
\begin{array}{lll}
F^{*}=F+\sum_{i=1}^{t} b_{i} D_{i} & \text { with } & \left(F^{*}, D_{\jmath}\right)=0 \\
G^{*}=G+\sum_{i=1}^{t} c_{i} D_{i} & \text { with } & \left(G^{*}, D_{\jmath}\right)=0
\end{array}
$$

for every $1 \leqq j \leqq t . \quad F^{*}$ and $G^{*}$ are uniquely determined. Since $K_{V}+D \equiv n F+G$, we have apparently $K_{V}+D^{*} \equiv n F^{*}+G^{*}$. We now prove the following :

Lemma 1.1. (1) We have $F^{*} \geqq F, 0 \leqq G^{*} \leqq G,\left(F^{*}\right)^{2} \geqq\left(F^{2}\right) \geqq 0$ and

$$
\left(K_{V}+D^{*}\right)^{2} \geqq n\left(K_{V}+D^{*}, F^{*}\right) \geqq n^{2}\left(F^{*}\right)^{2} .
$$

(2) Among irreducible components of $D^{*}$, let $C_{i}(1 \leqq i \leqq p)\left(r e s p . E_{\jmath}(1 \leqq j \leqq q)\right)$ exhaust the components meeting $F$ and with coefficient $\alpha_{i}<1$ (resp. $\alpha_{j}=1$ ). Then we have:

$$
\begin{gathered}
\left(K_{V}+D^{*}, F^{*}\right) \geqq\left(K_{V}, F\right)+\sum_{i=1}^{p}\left(1-\frac{2}{\gamma_{i}}\right)\left(C_{\imath}, F\right)+\sum_{j=1}^{q}\left(E_{\jmath}, F\right), \\
\left(F^{*}\right)^{2} \geqq \sum_{i=1}^{p} \frac{\left(C_{i}, F\right)^{2}}{\gamma_{i}}, \quad \text { and } \\
\left(K_{V}+D^{*}, F^{*}\right)+2\left(F^{*}\right)^{2} \geqq\left(K_{V}, F\right)+\sum_{i=1}^{p}\left(C_{\imath}, F\right)+\sum_{j=1}^{q}\left(E_{\jmath}, F\right) \geqq 1,
\end{gathered}
$$

where $\gamma_{i}:=-\left(C_{i}^{2}\right)$.

Proof. (1) Since $0=\left(F^{*}, D_{j}\right)=\left(F, D_{j}\right)+\sum_{\imath=1}^{t} b_{i}\left(D_{i}, D_{\jmath}\right) \geqq \sum_{i=1}^{t} b_{i}\left(D_{i}, D_{\jmath}\right)$ and since the intersection matrix $\left(\left(D_{i}, D_{\jmath}\right)\right)_{1 \leqq \imath, \jmath \leq t}$ is negative definite, every $b_{\imath}$ must be non-negative. Hence $F^{*} \geqq F$.

We write $G^{*}=\check{G}+\sum_{i=1}^{t} c_{i}^{\prime} D_{i}$ so that $\tilde{G}$ is an effective integral divisor and $\tilde{G}$ contains no components of $\operatorname{Supp}\left(D-D^{*}\right)$. Similarly as above, we can show that $c_{i}^{\prime} \geqq 0(0 \leqq i \leqq t)$ and $G^{*} \geqq \tilde{G} \geqq 0$. On the other hand, we have $G^{*}-G \equiv\left(K_{V}+\right.$ $\left.D^{*}-n F^{*}\right)-\left(K_{V}+D-n F\right) \equiv D^{*}-D+n\left(F-F^{*}\right)$. Write $D^{*}-D+n\left(F-F^{*}\right)-\left(G^{*}-\right.$ 
$G)=A-B$ such that $A$ and $B$ are effective $Q$-divisors with no common components. We have proved $A-B \equiv 0$. Note that $\operatorname{Supp}(B) \subseteq \operatorname{Supp}\left(D^{*}-D\right) \cup$ $\operatorname{Supp}\left(F-F^{*}\right) \cup \operatorname{Supp}\left(G^{*}-G\right)=\operatorname{Supp}\left(D-D^{*}\right)$. So, $B$ has negative definite intersection matrix. Hence we have $0=(A-B, B)=(A, B)-\left(B^{2}\right) \geqq-\left(B^{2}\right) \geqq 0$. Thus, $\left(B^{2}\right)=0$ and $B=0$. Then $A=A-B \equiv 0$ and $A=0$. So, $G^{*}-G=D^{*}-D+n\left(F-F^{*}\right)$ $\leqq D^{*}-D \leqq 0$.

Noting that $F, F^{*}$ and $K_{V}+D^{*}$ are nef divisors, we obtain :

and

$$
\left(F^{*}\right)^{2}=\left(F^{*}, F\right) \geqq\left(F^{2}\right) \geqq 0,
$$

$$
\left(K_{V}+D^{*}\right)^{2} \geqq n\left(K_{V}+D^{*}, F^{*}\right)=n\left(n F^{*}+G^{*}, F^{*}\right) \geqq n^{2}\left(F^{*}\right)^{2} .
$$

(2) We have

$$
\begin{aligned}
\left(\Sigma\left(\alpha_{\imath}-1+\frac{2}{\gamma_{\imath}}\right) C_{\imath}, C_{\jmath}\right) & =\left(K_{V}+\Sigma \alpha_{\imath} C_{\imath}, C_{\jmath}\right)-\left(K_{V}+\Sigma\left(1-\frac{2}{\gamma_{i}}\right) C_{\imath}, C_{\jmath}\right) \\
& \leqq\left(K_{V}+D^{*}, C_{\jmath}\right)-\left(K_{V}+\sum_{\imath \neq \jmath}\left(1-\frac{2}{\gamma_{i}}\right) C_{\imath}, C_{\jmath}\right) \leqq 0
\end{aligned}
$$

because $\left(K_{Y}+D^{*}, C_{i}\right)=0$ and $\left(K_{\mathrm{r}^{r}}, C_{i}\right) \geqq 0$. Indeed, since $\alpha_{i}<1$, we have $C_{i} \subseteq$ $\operatorname{Supp}\left(D-D^{*}\right)$ and $C_{\imath}$ is a nonsingular rational curve with $\gamma_{i}=-\left(C_{\imath}^{2}\right) \geqq 2$. Hence we obtain

$$
D^{*} \geqq \sum_{\imath=1}^{p} \alpha_{\imath} C_{\imath}+\sum_{j=1}^{q} E_{\jmath} \geqq \sum_{\imath=1}^{p}\left(1-\frac{2}{\gamma_{i}}\right) C_{\imath}+\sum_{j=1}^{q} E_{\jmath} .
$$

Similarly, we have

$$
F^{*}=F+\sum_{\imath=1}^{t} b_{i} D_{\imath} \geqq F+\sum_{\imath=1}^{p} \frac{\left(C_{\imath}, F\right)}{\gamma_{\imath}} C_{\imath} .
$$

Therefore, we have:

$$
\begin{aligned}
& \left(K_{V}+D^{*}, F^{*}\right)=\left(K_{V}+D^{*}, F\right) \\
& \quad \geq\left(K_{V}, F\right)+\sum_{i=1}^{p}\left(1-\frac{2}{\gamma_{\imath}}\right)\left(C_{\imath}, F\right)+\sum_{\jmath=1}^{q}\left(E_{\jmath}, F\right),
\end{aligned}
$$

and

$$
\left(F^{*}\right)^{2}=\left(F^{*}, F\right) \geqq\left(F+\sum_{i=1}^{p} \frac{\left(C_{i}, F\right)}{\gamma_{i}} C_{\imath}, F\right) \geqq \sum_{i=1}^{p} \frac{\left(C_{i}, F\right)^{2}}{\gamma_{\imath}}
$$

So, the inequalities (1.1b) and (1.1c) are obtained. Combining (1.1b) and (1.1c), we obtain the first half of $(1.1 \mathrm{~d})$. Since $K_{V}+D^{*}$ is nef and big and since $F$ moves in a pencil $\varphi: V \rightarrow \bar{B}$, we have $0<\left(K_{V}+D^{*}, F\right)=\left(K_{V}, F\right)+\Sigma \alpha_{i}\left(C_{\imath}, F\right)+$ $\Sigma\left(E_{\jmath}, F\right) \leqq\left(K_{V}, F\right)+\Sigma\left(C_{i}, F\right)+\Sigma\left(E_{\jmath}, F\right)$. Hence the second half of (1.1d) is proved.

Lemma 1.1 gives the following:

Proposition 1.2. Let $(V, D)$ be a minimal log surface of general type such 
that $\left|K_{V}+D\right|$ is composed with a pencil. Write $\left|K_{V}+D\right|=|C|+G$ and $C \equiv n F$ as above. Let $\alpha>0$ and $\beta$ be rational numbers such that $f(n ; \alpha, \beta)=(\alpha-1) n^{2}+$ $(\beta-3) n+2(\beta-1) \geqq 0$. Then $\bar{p}_{g} \leqq \alpha\left(K_{V}+D^{*}\right)^{2}+\beta$.

Proof. Let $\alpha>0$ and $\beta$ be rational numbers such that $\bar{p}_{g}>\alpha\left(K_{V}+D^{*}\right)^{2}+\beta$. By Lemma 1.1 and by noting that $\bar{p}_{g} \leqq n+1$, we have:

$$
\begin{aligned}
& 1 \leqq\left(K_{V}+D^{*}, F^{*}\right)+2\left(F^{*}\right)^{2} \leqq\left(\frac{1}{n}+\frac{2}{n^{2}}\right)\left(K_{V}+D^{*}\right)^{2} \\
&<\frac{(n+2)\left(\bar{p}_{g}-\beta\right)}{\alpha n^{2}} \leqq \frac{(n+2)(n+1-\beta)}{\alpha n^{2}}
\end{aligned}
$$

and $f(n ; \alpha, \beta)<0$.

Now we can verify Theorem 1.3.

By Lemma 1.1, we have:

$$
1 \leqq\left(K_{V}+D^{*}, F^{*}\right)+2\left(F^{*}\right)^{2} \leqq\left(\frac{1}{n}+\frac{2}{n^{2}}\right)\left(K_{V}+D^{*}\right)^{2},
$$

and thence

$$
n^{2}-\left(K_{V}+D^{*}\right)^{2} n-2\left(K_{V}+D^{*}\right)^{2} \leqq 0 .
$$

This implies:

$$
\left.\bar{p}_{g} \leqq n+1 \leqq \frac{1}{2}\left\{\left(K_{V}+D^{*}\right)^{2}+\sqrt{\left(\left(K_{V}+D^{*}\right)^{2}\right.}\right)^{2} \overline{+} \overline{\left(K_{V}+D^{*}\right)^{2}}\right\}+1 .
$$

The other two inequalities of Theorem 1.3 follow from (1.3c).

We shall give a sufficient condition for $\varphi: V \rightarrow \bar{B}$ to be an elliptic fibration or a $P^{1}$-fibration, where $\varphi$ is the same as defined before Lemma 1.1 .

Proposition 1.4. Let $(V, D)$ be a minimal log surface of general type such that $\mid K_{V}+D_{\mid}$is composed with a pencil. Suppose that one of the following conditions (1) and (2) is satisfied.

(1) $\bar{p}_{g} \geqq 1 / 2\left(K_{V}+D^{*}\right)^{2}+3$

(2) There are rational numbers $\alpha, \beta$ such that $\alpha>(1 / 2)+(\beta-3)^{2} / 16(\beta-1)=$ $(\beta+3 / 16)+(1 / 4(\beta-1)), \beta>1$ and $\bar{p}_{g} \geqq \alpha\left(K_{V}+D^{*}\right)^{2}+\beta$.

Then $\left(K_{V}, F\right)+\Sigma\left(C_{i}, F\right)+\Sigma\left(E_{j}, F\right)=1$ (cf. Lemma 1.1) and $\varphi: V \rightarrow \bar{B}$ is either an elliptic fibration or a $\boldsymbol{P}^{1}$-fibration.

Proof. Suppose $\bar{p}_{g} \geqq \alpha\left(K_{V}+D^{*}\right)^{2}+\beta$ for some rational numbers $\alpha \geqq 1 / 2$ and $\beta>1$. Then $\left(K_{V}+D^{*}\right)^{2} \leqq\left(\bar{p}_{g}-\beta\right) / \alpha \leqq(n+1-\beta) / \alpha$ because $\bar{p}_{g} \leqq n+1$. By Lemma 1.1 , we have $0 \leqq\left(F^{2}\right) \leqq\left(F^{*}\right)^{2} \leqq\left(K_{V}+D^{*}\right)^{2} / n^{2} \leqq(n+1-\beta) / \alpha n^{2}<1$ if $(\alpha, \beta)=(1 / 2,3)$ or if $\alpha>1 / 4(\beta-1)$. The last hypotheses are satisfied respectively by the conditions (1) and (2) above. Thence we have $\left(F^{2}\right)=0$, and hence $\Phi_{|C|}$ and $\varphi$ are morphisms. By virtue of Lemma 1.1 we have 


$$
\begin{aligned}
1 & \leqq\left(K_{V}, F\right)+\Sigma\left(C_{i}, F\right)+\Sigma\left(E_{\jmath}, F\right) \\
& \leqq\left(K_{V}+D^{*}, F^{*}\right)+2\left(F^{*}\right)^{2} \leqq\left(\frac{1}{n}+\frac{2}{n^{2}}\right)\left(K_{V}+D^{*}\right)^{2} \\
& \leqq\left(\frac{1}{n}+\frac{2}{n^{2}}\right)(n+1-\beta) / \alpha=(n+2)(n+1-\beta) / \alpha n^{2}<2
\end{aligned}
$$

if $(\alpha, \beta)=(1 / 2,3)$ or if $\alpha>(1 / 2)+(\beta-3)^{2} / 16(\beta-1)$. So, $\left(K_{V}, F\right)+\Sigma\left(C_{i}, F\right)+$ $\sum\left(E_{\jmath}, F\right)=1$ because the left hand side is an integer, and hence $2 g(F)-2=$ $\left(K_{V}, F\right) \leqq 1$ which implies $g(F) \leqq 1$. This completes the proof of Proposition 1.4.

The first assertion of the following lemma follows from the theory of peeling (cf. Miyanishi and Tsunoda [4; p. 438]).

Lemma 1.5. Let $(V, D)$ be a minimal log surface. Write $\left|K_{V}+D\right|=|C|+$ $G$ with the movable part $|C|$ and the fixed part $G$. Then the following assertions hold.

(1) Every connected component of Supp D-Supp $D^{*}$ is a connected component of $D$, consists of (-2)-curves and is contracted to a rational double singular point. More precisely, if $D-D^{*}=\sum_{\imath=1}^{t}\left(1-\alpha_{\imath}\right) D_{i}$ where $0 \leqq \alpha_{\imath}<1$, then $\operatorname{Supp} D-\operatorname{Supp} D^{*}$ $=\operatorname{Supp} \sum_{\alpha_{i}=0} D_{i}$.

(2) We have $G \geqq D-\left[D^{*}\right]$ and $\operatorname{Supp} G \supseteqq \operatorname{Supp}\left(D-\left[D^{*}\right]\right)=\operatorname{Supp}\left(D-D^{*}\right)$. Here $\left[D^{*}\right]$ is the largest effective integral divisor satisfying $D^{*} \geqq\left[D^{*}\right]$.

(3) $|C|$ and $G+\left[D^{*}\right]-D$ are respectively the movable part and the fixed part of $\left|K_{V}+\left[D^{*}\right]\right|$.

(4) $h^{0}\left(V, K_{V}+D\right)=h^{0}\left(V, K_{V}+\left[D^{*}\right]\right)$.

Proof. We have $G \geqq D-D^{*}$ because $\operatorname{Supp}\left(D-D^{*}\right)$ has negative definite intersection matrix and

$$
\left(G-\left(D-D^{*}\right), D_{\jmath}\right)=\left(K_{V}+D^{*}-C, D_{1}\right)=-\left(C, D_{1}\right) \leqq 0,
$$

for every irreducible component $D$, of $D-D^{*}$. Thence follow the assertions (2), (3) and (4).

If the first inequality of Theorem 1.3 becomes an equality for a pair $(V, D)$, its structure will be explicitly described in the subsequent propositions 1.6 and 1.7 .

Proposition 1.6. Let $(V, D)$ be a minimal log surface of general type such that $\left|K_{V}+D\right|$ is composed with a pencil and that $\bar{p}_{g}=1 / 2\left\{\left(K_{V}+D^{*}\right)^{2}+\right.$ $\sqrt{\left.\left(\left(K_{V}+D^{*}\right)^{2}\right)^{2}+8\left(K_{V}+D^{*}\right)^{2}\right\}}+1$. Then, with the above notations, the following assertions hold.

(1) All inequalities in (1.1a)-(1.1d) of Lemma 1.1 become equalities.

(2) We have $\left(F^{2}\right)=0$. IIence $\Phi_{|c|}: V \rightarrow \boldsymbol{P}^{\mathrm{V}}$ and $\varphi: V^{\top} \rightarrow \bar{B}$ are morphisms.

(3) We have $\bar{p}_{g}=N+1=n+1$. Hence $B=\bar{B} \cong \boldsymbol{P}^{1}$ and $B$ has degree $n$ in $\boldsymbol{P}^{n}$. 
(4) We have $g(F) \leqq 1$. Hence $\varphi$ is an elliptic fibration or a $\mathbb{P}^{1}$-fibration.

(5) Let $i: \boldsymbol{P}^{1} \rightarrow \mathbb{P}^{n}$ be the closed immersion defined by $\left|O_{P 1}(n)\right|$. Then $\Phi_{|C|}=i \cdot \varphi$.

(6) Let $C_{i}(1 \leqq i \leqq p)$ and $E_{j}(1 \leqq j \leqq q)$ be those components of $D^{*}$ defined in Lemma 1.1, (2). Then $C_{\imath}^{\prime}$ s are isolated components of $D$ and are cross-sections of $\varphi$. We have $p=1-\left(K_{V}, F\right)-\Sigma\left(E_{\jmath}, F\right) \leqq 1-\left(K_{V}, F\right) \leqq 3$.

(7) $\left(K_{V}+D^{*}\right)^{2}=n\left(K_{V}+D^{*}, F^{*}\right)=n^{2}\left(F^{*}\right)^{2}=n^{2} /(n+2)$.

Proof. Assume that the first inequality of Theorem 1.3 is an equality. Then the inequalities in (1.3a), (1.3b) and (1.3c) become equalities. Hence $\bar{p}_{g}=$ $n+1$ and the inequalities in (1.1a) and (1.1d) of Lemma 1.1 become equalities. Then the assertion(1) follows from the proof of Lemma 1.1 and the assertion (7) follows from (1.3b) and (1.1a).

By the assertion (1) and the proof of Lemma 1.1, we conclude that $\left(F^{2}\right)=0$, $\left(C_{\imath}, F\right)=1, p=\sum_{\imath=1}^{p}\left(C_{i}, F\right)=1-\left(K_{V}, F\right)-\sum\left(E_{\jmath}, F\right)$ and the coefficient $\alpha_{i}$ of $C_{\imath}$ in $D^{*}$ is equal to $1-\left(2 / \gamma_{i}\right)$ for $1 \leqq i \leqq p$, where $\gamma_{\imath}=-\left(C_{\imath}^{2}\right)$. So the $\operatorname{assertion}(2)$ follows. Therefore, $C_{i}$ does not meet components of $D^{*}$ other than $C_{i}$ because $\left(K_{V}+D^{*}, C_{i}\right)=0$. Hence $C_{i}^{\prime}$ s are isolated components of Supp $D^{*}$. Then $C_{i}^{\prime} s$ are isolated components of $D$ by Lemma 1.5, (1). This proves the assertion(6). Since $2 p_{a}(F)-2=\left(K_{V}, F\right) \leqq 1-p \leqq 1$, the assertion (4) follows.

Since we have $\bar{p}_{g}=n+1$, from the argument for the proof of $\bar{p}_{g}=N+1 \leqq$ $n+1$ before Lemma 1.1, we have $n=d e=d=\bar{p}_{g}-1=N$. So, $B$ is a curve of degree $d=n$ in $\mathbb{P}^{n}$ and $\bar{B} \rightarrow B$ is a birational morphism. Then we obtain the assertion(3) because every irreducible curve of degree $n$ in $\mathbb{P}^{n}$, which is not contained in any hyperplane of $\mathbb{P}^{n}$, is, upto a projective isomorphism, the image of $\boldsymbol{P}^{1}$ under the closed immersion $i: \mathbb{P}^{1} \rightarrow \mathbb{P}^{n}$ defined by $\left|\mathcal{O}_{P_{1}}(n)\right|$. This proves also the assertion (5). Indeed, note that $\varphi^{*} i^{*} \mathcal{O}_{P n}(1) \cong \varphi^{*} \mathcal{O}_{P 1}(n) \cong \mathcal{O}_{V}(n F) \cong \mathcal{O}_{V}(C)$.

Proposition 1.7. With the same assumptions and notations as in Proposition 1.6, we have the following assertions:

(1) We have $p=1$ and $\left(C_{1}^{2}\right)=-n-2$. Hence $\sum_{j=1}^{q}\left(E_{\jmath}, F\right)=-\left(K_{V}, F\right)(c f$. Proposition 1.6, (6)).

(2) $\operatorname{Supp}\left(D-D^{*}\right)$ consists of several connected components of $D$. More precisely, we have $D-D^{*}=(2 / n+2) C_{1}+\sum_{k=2}^{t} D_{k}$, where Supp $\sum_{k=2}^{t} D_{k}=\operatorname{Supp} D-$ Supp $D^{*}$ (cf. Lemma 1.5, (1)).

(3) Let $D^{\prime}$ be the reduced effective divisor consisting of all components of $D^{*}$ with coefficient 1 . Then $D=D^{\prime}+C_{1}+\sum_{k=2}^{\iota} D_{k}$ and $D^{*}=D^{\prime}+(n / n+2) C_{1}$. Moreover, $K_{V}+D^{\prime} \sim n F, G=D-D^{\prime}$ and $F^{*}=F+(1 / n+2) C_{1}$.

Proof. We prove first $G^{*}=0$ which will imply $\operatorname{Supp} G=\operatorname{Supp}\left(D-D^{*}\right)$ by the definition of $G^{*}$ and by Lemma 1.5, (2). By Proposition 1.6, (1) and by the proof of Lemma 1.1, (1.1a), we have: 


$$
0=\left(K_{V}+D^{*}, G^{*}\right)=\left(G^{*}, F^{*}\right)\left(=\left(G^{*}, F\right)\right) .
$$

Hence $G^{*}$ is contained in fibers of $\varphi$. On the other hand, $\left(G^{*}\right)^{2}=\left(G^{*}, K_{V}+D^{*}-\right.$ $\left.n F^{*}\right)=0$. Hence $G^{*} \equiv \alpha F$ with a rational number $\alpha$. Then $0=\left(G^{*}, F^{*}\right)=\alpha\left(F, F^{*}\right)$ $=\alpha\left(F^{*}\right)^{2}$ and hence $\alpha=0$ because $\left(F^{*}\right)^{2}>0$. So, $G^{*} \equiv 0$. This, together with the fact $G^{*} \geqq 0$, implies $G^{*}=0$.

Write $D-D^{*}=\sum_{i=1}^{p}\left(1-\alpha_{i}\right) C_{i}+\sum_{k=p+1}^{t}\left(1-\alpha_{k}\right) D_{k}$, where $0 \leqq \alpha_{j}<1$ for $1 \leqq j \leqq t$. By Proposition 1.6, (1) and by the proof of Lemma 1.1, (1.1b), we have $\alpha_{i}=1$ $\left(2 / \gamma_{i}\right)$ where $\gamma_{i}=-\left(C_{\imath}^{2}\right)(1 \leqq i \leqq p)$. Write $F^{*}=F+\sum_{i=1}^{p} b_{i} C_{i}+\sum_{k=p+1}^{t} b_{k} D_{k}$. Similarly, we have $b_{i}=\left(C_{i}, F\right) / \gamma_{i}=1 / \gamma_{i}(1 \leqq i \leqq p)$ and $1=(n+2)\left(F^{*}\right)^{2}=(n+2) \sum_{i=1}^{p}\left(C_{i}\right.$, $F)^{2} / \gamma_{i}=\sum_{i=1}^{p}(n+2) / \gamma_{i}$ (cf. Proposition 1.6, (7)). In particular, $p \geqq 1$.

We claim that if $p+1 \leqq k \leqq t$ and if $\alpha_{k}>0$, then $b_{k}=0$. Indeed, suppose $\alpha_{k}>0$. Then $D_{k} \leqq \operatorname{Supp} D^{*}$. Hence $\left(D_{k}, F\right)=0$ by Proposition $1.6,(6)$ and by noting that $\alpha_{k}<1$. This, together with $\left(D_{k}, C_{i}\right)=0$ (cf. Proposition 1.6, (6)), implies $0=\left(\sum_{n_{k}>0} b_{k} D_{k}, F^{*}\right)=\left(\sum_{\alpha_{k}>0} b_{k} D_{k}, \sum b_{k} D_{k}\right)=\left(\sum_{n_{k}>0} b_{k} D_{k}\right)^{2}$ by Lemma 1.5 , (1). Hence the claim is true because $\sum D_{k}$ has negative definite intersection matrix.

Note that $G=G-G^{*} \equiv K_{V}+D-n F-\left(K_{V}+D^{*}-n F^{*}\right) \equiv n\left(F^{*}-F\right)+D-D^{*} \equiv$ $\Sigma\left(n+2 / \gamma_{i}\right) C_{i}+\sum\left(n b_{k}+1-\alpha_{k}\right) D_{k}$. Since $\operatorname{Supp}\left(G+\Sigma C_{i}+\sum D_{k}\right)\left(=\operatorname{Supp}\left(D-D^{*}\right)\right)$ has negative definite intersection matrix, we have $G=\Sigma\left(n+2 / \gamma_{i}\right) C_{i}+\Sigma\left(n b_{k}+\right.$ $\left.1-\alpha_{k}\right) D_{k} \leqq \sum C_{i}+\sum_{a_{k}=0}\left(n b_{k}+1\right) D_{k}+\sum_{\alpha_{k}>0}\left(1-\alpha_{k}\right) D_{k}$. Since $G$ is integral and since $\alpha_{k}<1$, we see that $p=1, \alpha_{k}=0(2 \leqq k \leqq t)$ and $-\left(C_{1}^{2}\right)=\gamma_{1}=n+2$. In particular, the assertion (1) is true. We have also $D-D^{*}=(2 / n+2) C_{1}+\sum_{k=2}^{t} D_{k}$ and Supp $\sum_{k=2}^{t} D_{k}=\operatorname{Supp} \sum_{a_{k}=0} D_{k}=\operatorname{Supp} D-\operatorname{Supp} D^{*}$. Thus, the assertion (2) is proved.

Now we shall prove the assertion(3). The first part follows from (2). We assert that $\sum b_{k} D_{k}=0$. Indeed, $\left(\sum b_{k} D_{k}\right)^{2}=\left(F^{*}-F-(1 / n+2) C_{1}\right)^{2}=\left(F^{*}\right)^{2}+\left(C_{1}^{2}\right) /$ $(n+2)^{2}-2\left(F^{*}, F\right)+2\left(F,(1 / n+2) C_{1}\right)=(1 / n+2)-(1 / n+2)-2\left(F^{*}\right)^{2}+(2 / n+2)=0$. Hence $\Sigma b_{k} D_{k}=0$ because $\Sigma D_{k}$ has negative definite intersection matrix. Thus, $F^{*}=F+(1 / n+2) C_{1}, G=C_{1}+\sum_{k=2}^{t} D_{k}=D-D^{\prime}$ and $K_{r^{r}}+D^{\prime}=K_{V}+D-G \sim n F$.

We end this section with the following example. In view of this example, the bounds for $\bar{p}_{g}$ in Theorem 1.3 are the best possible ones.

Example 1.8. Let $\varphi: V \rightarrow \boldsymbol{P}^{1}$ be a relatively minimal elliptic fibration having a cross-section $D$ with $s:=-\left(D^{2}\right) \geqq 2$. Then $K_{V} \sim(s-2) F$ with a general fiber $F$ of $\varphi$. Note that $D$ is the fixed part of $\left|K_{V}+D\right|$ and $K_{V}+D^{*}=K_{V}+(s-2 / s) D$. Hence $\bar{p}_{g}=p_{g}(V)=s-1$ and $\left(K_{V}+D^{*}\right)^{2}=(s-2)^{2} / s$. In particular, if $s=6$, then $(V, D)$ is a minimal log surface of general type satisfying $\bar{p}_{g}=p_{g}(V)=(9 / 8)\left(K_{V}+\right.$ $\left.D^{*}\right)^{2}+2$. We shall show below by giving examples that $s$ can be arbitrarily large. Hence, if we write $\bar{p}_{g} \leqq \alpha\left(K_{V}+D^{*}\right)^{2}+\beta$, we have

$$
s(s-1) \leqq \alpha(s-2)^{2}+\beta s .
$$

Then bringing $s$ to $\infty$, we know that $\alpha \geqq 1$, and if we take $\alpha=1$ then $\beta \geqq 3$. 
We construct a relatively minimal elliptic surface $\varphi: V \rightarrow \mathbb{P}^{1}$ as above with $s:=-\left(D^{2}\right)$ as large as we wish. Let $\Sigma_{2 s}$ be a Hirzebruch surface of degree $2 s$ with $s \geqq 0$, let $M_{2 s}$ be the minimal section of self-intersection number $-2 s$ and let $L$ be a general fiber of a $\boldsymbol{P}^{1}$-fibration $\pi: \Sigma_{2 s} \rightarrow \boldsymbol{P}^{1}$. Let $C_{1}, C_{2}$ and $C_{3}$ be cross-sections linearly equivalent to $M_{2 s}+2 s L$ such that $C_{1}, C_{2}$ and $C_{3}$ have no common points. Let $\sigma: W \rightarrow \sum_{2 s}$ be a double covering with branch locus $M_{2 s}+$ $C_{1}+C_{2}+C_{3}\left(\sim 4 M_{2 s}+6 s L\right)$ and let $\tau: V \rightarrow W$ be the minimal resolution of singularities. By the construction, $W$ has only rational double singular points of Dynkin type $A_{1}$. In fact, let $P$ be a common point of $C_{1}$ and $C_{2}$. Then $P$ is not lying on $C_{3}$. Blow up the point $P$ to get a $(-1)$-curve $E$. Let $L_{1}$ be a fiber of $\pi$ passing through $P$ and let $L_{1}^{\prime}$ be the proper transform of $L_{1}$. Then the inverse image of $E+L_{1}^{\prime}$ on $V$ is a union of two (-2)-curves meeting each other in two distinct points, which is a degenerate fiber of the elliptic fibration $\varphi$ : $V \rightarrow \boldsymbol{P}^{1}$, which is induced from $\pi$. Thus the elliptic fibration $\varphi$ is relatively minimal. Let $\tau^{*} \sigma^{*}\left(M_{2 s}\right)=2 D$. Then $D$ is a cross-section of $\varphi$ with $\left(D^{2}\right)=-s$.

\section{$\S 2$. The Case where $\left|K_{V}+D\right|$ is not Composed with a Pencil}

Let $(V, D)$ be a minimal surface of general type such that $\bar{p}_{g} \geqq 3$ and the movable part $|C|$ of $\left|K_{V}+D\right|$ is not composed with a pencil. Then $\Phi_{|C|}(V)$ is a surface in $\boldsymbol{P}^{N}$, where $N:=\bar{p}_{g}-1=h^{0}(V, C)-1$. Hence, by the Bertini theorem, every general member of $|C|$ is a reduced irreducible curve with positive self-intersection number. We shall consider, in general, a curve $A$ on a nonsingular projective surface $V$ satisfying:

(*) $\quad h^{0}(V, A) \geqq 3$ and $|A|$ is not composed with a pencil.

In the subsequent arguments, we always assume that $C$ and $A$ are general members of $|C|$ and $|A|$, respectively. We are going to prove Theorem 2.8 which will imply easily Theorem 2.10 . We need several preliminary results which give several inequalities of the form $h^{0}(V, A) \leqq \alpha\left(A^{2}\right)+\beta$ with $\alpha, \beta \in Q$ under certain conditions.

Since $A$ is an irreducible curve and since $h^{0}(V, A) \geqq 3,|A|$ contains no fixed components. If $x_{1}$ is a base point of $|A|$, let $m_{1}$ be the multiplicity of the curve $A$ at $x_{1}$ and let $f_{1}: V_{1} \rightarrow V$ be the blowing-up of the point $x_{1}$. If $x_{2}$ is a base point of $\left|f_{1}^{\prime}(A)\right|$, let $m_{2}$ be the multiplicity of the curve $f_{1}^{\prime}(A)$ at $x_{2}$ and let $f_{2}: V_{2} \rightarrow V_{1}$ be the blowing-up of the point $x_{2}$. Continue this process. Then, at the $b$-th step for some $b \geqq 0,\left|f^{\prime}(A)\right|$ is base point free. Here we set $W=V_{b}$ and $f:=f_{b} \cdots f_{1}: W \rightarrow V$.

Lemma 2.1. Assume that $A$ satisfies the above condition (*). Then the following assertions hold.

(1) $h^{0}(V, A)=h^{0}\left(W, f^{\prime}(A)\right) \leqq\left(f^{\prime}(A)\right)^{2}+2=\left(A^{2}\right)+2-\sum_{2=1}^{b} m_{2}^{2}$. 
(2) If $h^{0}(V, A)=\left(A^{2}\right)+2$, then $\Phi_{|A|}$ is a birational morphism onto a surface $\bar{W}$ of degree $N-1$ in $\boldsymbol{P}^{N}$. Here $N:=h^{\circ}(V, A)-1$.

Proof. By the definition of $f$, we have $3 \leqq h^{0}(V, A)=h^{0}\left(W, f^{\prime}(A)\right)$ and $1 \leqq$ $\left(f^{\prime}(A)\right)^{2}=\left(A^{2}\right)-\sum_{i=1}^{b} m_{i}^{2}$. In order to prove (1), we have only to consider the case where $|A|$ is base point free. Then every general member $A_{1}$ of $\mid A$ is nonsingular and irreducible by the Bertini theorem. Replace $A$ by a general member. Let $h=h_{\left(A^{2}\right)} \cdots h_{1}: V^{\prime} \rightarrow V$ be a composite of blowing-ups of all $\left(A^{2}\right)$ intersection points of $A$ and $A_{1}$ such that $h^{\prime}(A) \cap h^{\prime}\left(A_{1}\right)=\phi$. Then $h^{\prime}(A) \sim h^{\prime}\left(A_{1}\right)$, $\operatorname{dim}\left|h^{\prime}(A)\right|=1$ and we have a fibration $\Phi_{\left|h^{\prime}(A)\right|}: V^{\prime} \rightarrow \boldsymbol{P}^{1}$. Note that $\operatorname{dim} \mid\left(h_{i} \cdots\right.$ $\left.h_{1}\right)^{\prime}(A)|\geqq \operatorname{dim}|\left(h_{i-1} \cdots h_{1}\right)^{\prime}(A) \mid-1$ for each $i$. Then (1) follows.

Suppose $h^{0}(V, A)=\left(A^{2}\right)+2$. Then $|A|$ is base point free by (1). Note that $\bar{W}:=\Phi_{|A|}(V)$ is a surface in $\boldsymbol{P}^{N}\left(N:=h^{0}(V, A)-1\right)$ and is not contained in any hyperplane. Hence we have $d:=\operatorname{deg} \bar{W} \geqq \operatorname{codim} \bar{W}+1$. Namely, we have $d \geqq$ $N-1$. Then $\operatorname{deg} \Phi_{|A|}=\left(A^{2}\right) / d \leqq\left(A^{2}\right) /(N-1)=1$. Hence $\Phi_{|A|}$ is a birational morphism.

Lemma 2.1, (1) implies that $h^{0}(V, A) \leqq\left(A^{2}\right)+2$. This inequality has been proved by Sakai (cf. [6; Theorem 6.5]). $\vec{W}$ is described in the following Lemma 2.2 (cf. Nagata [5; Theorem 7]).

Lemma 2.2. If an irreducible surface $\bar{W}$ of degree $N-1$ in $\boldsymbol{P}^{N}$ is not contained in any hyperplane of $\boldsymbol{P}^{N}$, then $\bar{W}$ is one of the following:

(1) $\quad N=2$ and $\bar{W}=\boldsymbol{P}^{2}$.

(2) $\quad N=5$ and $\bar{W}=\boldsymbol{P}^{2}$ embedded in $\boldsymbol{P}^{5}$ by $\left|\mathcal{O}_{\boldsymbol{P}^{2}}(2)\right|$.

(3) $N=3,4,5, \cdots, \bar{W}=\sum_{e}$ where $0 \leqq N-e-3 \equiv 0(\bmod 2)$, and $\bar{W}$ is embedded in $\boldsymbol{P}^{N}$ by $\left|M_{e}+(1 / 2)(N+e-1) L\right|$.

(4) $N=3,4,5, \cdots$, and $\bar{W}$ is a cone over a rational curve of degree $N-1$ in $\boldsymbol{P}^{N-1}$.

Here $\Sigma_{e}$ is a Hirzebruch surface of degree $e, L$ is a fiber of a $\boldsymbol{P}^{1}$-fibration $\pi: \sum_{e} \rightarrow \boldsymbol{P}^{1}$ and $M_{e}$ is a minimal section with $\left(M_{e}^{2}\right)=-e$. In particular, $\bar{W}$ is $a$ normal rational surface.

We consider the case $h^{0}(V, A)=\left(A^{2}\right)+2$. Suppose that there is a $(-1)$-curve $E$ on $V$ satisfying $(A, E)=0$. Let $\tau_{1}: V \rightarrow W_{1}$ be the blowing-down of $E$ and let $\Gamma_{1}:=\tau_{1 *}(A)$. Then we have $A=\tau_{1}^{*}\left(\Gamma_{1}\right)$ and $\Phi_{|A|}=\Phi_{\left|\Gamma_{1}\right|} \cdot \tau_{1}$. Hence $\Gamma_{1}$ is nef and big because so is $A$. Continuing this process, we obtain a composite $\tau: V \rightarrow W$ of blowing-downs such that each (-1)-curve on $W$ has positive intersection number with $\Gamma:=\tau_{*}(A)$ and the following hold:

$$
A=\tau^{*}(\Gamma) \text { and } \Phi_{\mid A !}=\Phi_{|\Gamma|} \cdot \tau \text {. }
$$

Let $\Sigma$ be the reduced effective divisor consisting of all curves $E$ of $W$ satisfying $(E, \Gamma)=0$. Then $\Sigma$ contains no $(-1)$-curves. Let $\Sigma_{i}(1 \leqq i \leqq s)$ be all 
connected components of $\Sigma$. Note that $P_{i}:=\Phi_{1} \Gamma_{1}\left(\sum_{i}\right)$ is a single point, and each curve on $W$, which is contracted by $\Phi_{|\Gamma|}$, is contained in $\Sigma$. Since $\Phi_{|\Gamma|}$ is a birational morphism and since $\bar{W}=\Phi_{\Gamma_{1} 1}(W)$ is a normal surface, we have $P_{i} \neq P_{\jmath}(i \neq j)$ and $\Phi_{\mid \Gamma_{1}}: W-\Sigma \cong \bar{W}-\cup P_{i}$ by Zariski's Main Theorem. Hence $\Phi_{|\Gamma|}$ is a minimal resolution of singularities of $\bar{W}$.

Since $\mathcal{O}(\Gamma)=\Phi_{|\Gamma|}^{*} \mathcal{O}_{P} N(1)$, we obtain the following lemma by Lemmas 2.1 and 2.2, which has been obtained by Fujita and Sakai, independently (cf. Fujita [1; Theorem 3.2] and Sakai [6; Theorem 6.7]).

Lemma 2.3. Assume that $A$ satisfies the above condition (*) and assume further that $h^{\circ}(V, A)=\left(A^{2}\right)+2$. Let $\Gamma=\tau_{*}(A)$ and $V \stackrel{\tau}{\rightarrow} W \stackrel{\Phi_{\mid \Gamma 1}}{\longrightarrow} \bar{W} \leqq \mathbb{P}^{N}$ be the same as defined above. Then

$$
A=\tau^{*}(\Gamma), \quad \Phi_{|A|}=\Phi_{|\Gamma|} \cdot \tau,
$$

and $(W, \Gamma)$ is one of the following:

(1) $\left(\boldsymbol{P}^{2}, H\right)$, where $H$ is a hyperplane.

(2) $\left(\boldsymbol{P}^{2}, Q\right)$, where $Q \in\left|\boldsymbol{O}_{P 2}(2)\right|$.

(3) $\left(\Sigma_{e}, M_{e}+(1 / 2)(N+e-1) L\right)$, where $0 \leqq N-e-3 \equiv 0(\bmod 2)$.

(4) $\left(\Sigma_{N-1}, M_{N-1}+(N-1) L\right)$.

For the case $B s|A| \neq \phi$, we have:

Lemma 2.4. Assume that $A$ satisfies the above condition(*) and assume further that $V$ is not a rational surface and $|A|$ has base points. Then we have:

(1) $h^{0}(V, A) \leqq\left(A^{2}\right)$.

(2) Suppose $h^{\circ}(V, A)=\left(A^{2}\right)$. Then $A$ is nonsingular, $|A|$ has only one base point $P$ and $\left|h^{\prime}(A)\right|$ is base point free. Here $h: W \rightarrow V$ is the blowing-up of $P$.

Proof. We use the notations $f: W \rightarrow V$ and $\sum_{i=1}^{b} m_{\imath}^{2}$ in Lemma 2.1. We have $h^{0}\left(W, f^{\prime}(A)\right) \leqq\left(f^{\prime}(A)\right)^{2}+2$, and if the equality holds then $\Phi_{\left|f^{\prime}(A)\right|}$ is a birational morphism onto a rational surface in $\boldsymbol{P}^{N}\left(N:=h^{0}\left(W, f^{\prime}(A)\right)-1\right)$ by Lemma 2.2. Since $V$ is not a rational surface by the assumption, we have $h^{0}(V, A)=$ $h^{0}\left(W, f^{\prime}(A)\right) \leqq\left(f^{\prime}(A)\right)^{2}+1=\left(A^{2}\right)+1-\sum_{i=1}^{b} m_{i}^{2} \leqq\left(A^{2}\right)$ because $B s|A| \neq \phi$ by the assumption. This proves (1).

Suppose $h^{0}(V, A)=\left(A^{2}\right)$. Then $b=m_{1}=1$ and the assertion(2) follows with $h:=f$.

The case $p_{a}(A)=1$ is treated in Proposition 2.5. We shall use the following notation. Let $\pi: \boldsymbol{P}(E) \rightarrow B$ be a relatively minimal ruled surface defined by a normalized rank two vector bundle $E$ on a nonsingular curve $B$. We define the invariant $e(\boldsymbol{P}(E))=-\operatorname{deg} \wedge^{2} E$ as in Hartshorne [2; p. 373].

Proposition 2.5. Assume that $A$ satisfies the above condition $\left(^{*}\right)$ and assume further that $p_{a}(A)=1$ and $V$ is not a rational surface. Then the following as- 
sertions hold:

(1) We have $h^{0}(V, A)=\left(.4^{2}\right) . \quad V$ is such an elliptic ruled surface $\pi: V \rightarrow B$ that general members of $|A|$ are cross-sections of the $\boldsymbol{P}^{1}$-fibration $\pi$.

(2) There is a suitable composite $\tau: V \rightarrow W$ of blowing-downs of (-1)-curves in singular fibers of $\pi$ such that $W$ is a relatively minimal ruled surface and $A \sim$ $\tau^{*} \tau_{*}(A)$.

(3) The invariant $e=e(W)$ satisfies $4 \leqq\left(A^{2}\right)-e \equiv 0(\bmod 2)$.

(4) $\Phi_{(\tau(A),}: W \rightarrow \boldsymbol{P}^{2}$ is a finite morphism of degree three if $\left(A^{2}\right)=3, \Phi_{|\tau(A)|}$ : $W \rightarrow \boldsymbol{P}^{3}$ is a finite morphism of degree one (resp. or two) onto a quartic surface (resp. or quardratic surface) if $\left(A^{2}\right)=4$, and $\Phi_{|\tau(A)|}: W \rightarrow \Phi_{|\tau(A)|}(W)$ is the normalization morphism if $\left(A^{2}\right) \geqq 5$.

Proof. We shall use the arguments of Lemma 2.1. Let $\sigma_{1}: V_{1} \rightarrow V$ be a composite of blowing-ups of base points of $|A|$ so that $\left|\sigma_{1}^{\prime}(A)\right|$ is base point free $\left(\sigma_{1}=f\right.$ in the notation of Lemma 2.1). Let $\sigma_{2}: V_{2} \rightarrow V_{1}$ be a composite of blowing-ups so that the morphism $\Phi_{2}:=\Phi_{\left|\sigma_{2}^{\prime} \sigma_{1}^{\prime}(A)\right|}: V_{2} \rightarrow \boldsymbol{P}^{1}$ is a fibration $\left(\sigma_{2}=h\right.$ in the notation of Lemma 2.1). Since $V$ is not a rational surface and since $p_{a}(A)=1, A$ is an elliptic curve and $\Phi_{2}$ is an elliptic fibration. The last exceptional curve $E$ of $\sigma_{2}$ is a (-1)-curve and is a cross-section of $\Phi_{2}$. Let $\sigma_{3}$ : $V_{2} \rightarrow V_{3}$ be a composite of blowing-downs of (-1)-curves in singular fibers of $\Phi_{2}$ such that there are no (-1)-curves in any fiber of $\Phi_{3}:=\Phi_{2} \cdot \sigma_{3}^{-1}: V_{3} \rightarrow \boldsymbol{P}^{1}$. Then we have:

$$
\begin{aligned}
& \left(\sigma_{3}(E), K_{V_{3}}\right) \sigma_{3} \sigma_{2}^{\prime} \sigma_{1}^{\prime}(A) \sim K_{V_{3}} \\
& \sim\left(\operatorname{deg} K_{P 1}+\chi\left(\mathcal{O}_{V_{3}}\right)\right) \sigma_{3} \sigma_{2}^{\prime} \sigma_{1}^{\prime}(A) .
\end{aligned}
$$

Since $\left(\sigma_{3}(E), K_{V_{3}}\right)=-2-\left(\sigma_{3}(E)\right)^{2} \leqq-1, V_{3}$ is a ruled surface satisfying $q=q\left(V_{3}\right) \geqq 1$ and $-2-\left(\sigma_{3}(E)\right)^{2}=-2+\chi\left(\Theta_{V_{3}}\right)=-1-q$. Let $\pi_{3}: V_{3} \rightarrow B$ be the $\boldsymbol{P}^{1}$-fibration. In general, we have $\left(K_{V^{\prime}}^{2}\right) \leqq 8(1-q)$, and the equality holds if and only if $V_{3}$ is a relatively minimal ruled surface. In the present case, $\left(K_{V_{3}}^{2}\right)=0$. Hence $q=1$, $\left(\sigma_{3}(E)\right)^{2}=0$ and $V_{3}$ is a relatively minimal ruled surface. Evidently, $\sigma_{3}(E)$ is a fiber of $\pi_{3}$. Hence $\sigma_{3} \sigma_{2}^{\prime} \sigma_{1}^{\prime}(A)$ (resp. $\sigma_{2}^{\prime} \sigma_{1}^{\prime}(A)$, or $A$ ) is a cross-section of the $\boldsymbol{P}^{1}$. fibration $\pi_{3}$ (resp. $\pi_{2}:=\pi_{3} \cdot \sigma_{3}: V_{2} \rightarrow B$, or $\pi:=\pi_{3} \cdot \sigma_{3} \cdot \sigma_{2}^{-1} \cdot \sigma_{1}^{-1}: V \rightarrow B$ ). Let $\tau$ : $V \rightarrow W$ be a composite of blowing-downs of $(-1)$-curves, which are contained in singular fibers of $\pi$ and which do not meet $A$, such that $\tilde{\pi}:=\pi \cdot \tau^{-1}: W \rightarrow B$ is a relatively minimal ruled surface. Then $A=\tau^{*} \tau_{*}(A)=\tau^{*} \tau(A)$. The assertion (2) and the second part of the assertion (1) are proved.

Set $e=e(W)$. Then we have (cf. Hartshorne [2; p. 385]):

(1) $|\tau(A)|$ is base point free if and only if $(\tau(A))^{2}\left(=\left(A^{2}\right)\right) \geqq e+4$. If this is the case, $\tau(A)$ is ample and $\Phi_{|\tau(A)|}$ is a finite morphism because $|A|$ is not composed with a pencil.

(2) $\Phi_{: \tau(A) \mid}$ is a closed embedding if and only if $\left(A^{2}\right) \geqq e+6$.

Let $M_{e}$ be a minimal section of $W$ which has $\left(M_{e}^{2}\right)=-e$. We know that 
$0 \leqq\left(\tau(A), M_{e}\right)=(1 / 2)\left(\left(A^{2}\right)-e\right)$. Hence $0 \leqq\left(A^{2}\right)-e \equiv 0(\bmod 2)$. Write $\tau(A) \sim M_{e}+H$ where $H$ is the pullback of a divisor $h$ on $B$ with $\operatorname{deg} h=(1 / 2)\left(\left(A^{2}\right)+e\right)$. Consider an exact sequence:

$$
0 \longrightarrow \mathcal{O}_{V}(H) \longrightarrow \mathcal{O}_{V}(\tau(A)) \longrightarrow \mathcal{O}_{M_{e}}\left(\left.\tau(A)\right|_{M_{e}}\right) \longrightarrow 0 \text {. }
$$

Note that $e \geqq-1$ (cf. Hartshorne [2; Theorems 2.12 and 2.15, pp. 376-377]). We have $\operatorname{deg} h \geqq 0$ and $\left.\operatorname{deg} \tau(A)\right|_{M_{e}}=(1 / 2)\left(\left(A^{2}\right)-e\right) \geqq 0$. If $\operatorname{deg} h=0$ then $\left(A^{2}\right)=$ $-e=1,\left.\operatorname{deg} \tau(A)\right|_{M_{e}}=1$ and $h^{0}(V, A) \leqq h^{0}(V, H)+h^{0}\left(M_{e},\left.\tau(A)\right|_{M_{e}}\right)=h^{0}(B, h)+$ $\chi\left(\mathcal{O}_{M_{e}}(\tau(A))\right)=h^{0}(B, h)+\left.\operatorname{deg} \tau(A)\right|_{M_{e}} \leqq 1+1$. This contradicts the hypothesis. Therefore, $\operatorname{deg} h \geqq 1$. Then $H^{1}(V, H) \cong H^{1}(B, h) \cong H^{0}(B,-h)=0$ and $h^{0}(V, H)=$ $\operatorname{deg} h$. We have also $0 \leqq h^{0}\left(M_{e},-\left.\tau(A)\right|_{M_{e}}\right)=h^{0}\left(M_{e},\left.\tau(A)\right|_{M_{e}}\right)-\left.\operatorname{deg} \tau(A)\right|_{M_{e}} \leqq 1$ and the last inequality becomes an equality if and only if $\left.\tau(A)\right|_{M_{e}} \sim 0$. Then we have:

$$
\left(A^{2}\right) \leqq h^{0}(V, H)+h^{0}\left(M_{e},\left.\tau(A)\right|_{M_{e}}\right)=h^{0}(V, A) \leqq\left(A^{2}\right)+1,
$$

and $h^{0}(V, A)=\left(A^{2}\right)+1$ if and only if $\left.\tau(A)\right|_{M_{e}} \sim 0$. If $h^{0}(V, A)=\left(A^{2}\right)+1$, then $0=\left.\operatorname{deg} \tau(A)\right|_{M_{e}}=(1 / 2)\left(\left(A^{2}\right)-e\right)$ and $1 \leqq(\tau(A))^{2}=e<e+4$. Hence $|\tau(A)|$ has base point which leads to $h^{0}(V, A) \leqq\left(A^{2}\right)$ by Lemma 2.4. This is a contradiction. Therefore, we must have $h^{0}(V, A)=\left(A^{2}\right)$. The assertion (1) is proved.

Note that (3) is equivalent to saying that $|\tau(A)|$ is base point free. Suppose $|\tau(A)|$ has base points. Then $\left(A^{2}\right)=e$ or $e+2$. In view of Lemma $2.4,|\tau(A)|$ has only one base point $P$ and $\left|f^{\prime} \tau(A)\right|$ is base point free. Here $f: W_{1} \rightarrow W$ is the blowing-up of $P$. Let $L_{1}$ be the fiber of $\tilde{\pi}$ containing $P$ and let $g: W_{1} \rightarrow W_{2}$ be the blowing-down of the (-1)-curve $f^{\prime}\left(L_{1}\right)$. Then $g^{*} g_{*} f^{\prime} \tau(A)=f^{\prime} \tau(A)$ and $H^{0}\left(W_{2}, g_{*} f^{\prime} \tau(A)\right) \cong H^{0}\left(W_{1}, f^{\prime} \tau(A)\right) \cong H^{0}(W, \tau(A))$. Note that $\mid f^{\prime} \tau(A) !$ and $\mid g_{*} f^{\prime} \tau(A) !$ are base point free. Hence we must have $\left(g_{*} f^{\prime} \tau(A)\right)^{2} \geqq e\left(W_{2}\right)+4$. Since $\left(g_{*} f^{\prime} \tau(A)\right)^{2}=(\tau(A))^{2}-1 \leqq e+1$, we obtain $-1 \leqq e\left(W_{2}\right) \leqq e-3$ and $e \geqq 2$. In particular, $A \neq M_{e}$. On the other hand, $\left(M_{e}, L_{1}\right)=1$ implies $\left(g_{*} f^{\prime}\left(M_{e}\right)\right)^{2} \leqq\left(M_{e}^{2}\right)+$ $1=-e+1 \leqq-1$. Hence $g_{*} f^{\prime}\left(M_{e}\right)$ is equal to the minimal section $M_{e\left(W_{2}\right)}$ of $W_{2}$ and $e\left(W_{2}\right) \geqq e-1$. This contradicts the above inequality $e\left(W_{2}\right) \leqq e-3$. Therefore, $|\tau(A)|$ is base point free.

The assertion(4) can be proved similarly as in Lemma 2.1 .

We now consider the case where the geometric genus $g(A) \geqq 2$. We shall use the following Clifford theorem (cf. Martens $[3 ; \S 2.31]$ ).

Lemma 2.6. Let $X$ be a nonsingular projective curve of genus $g \geqq 2$ and let $L$ be a divisor on $X$ satisfying $0 \leqq \operatorname{deg} L \leqq 2 g-2$. Then the Clifford's index $c(L)$ : $=\operatorname{deg} L+2\left(1-h^{0}(X, L)\right) \geqq 0$ and the equality holds only if $L \sim 0, L \sim K_{X}$ or $X$ is a hyperelliptic curve.

Lemma 2.7. Assume that $A$ satisfies the above condition $\left(^{*}\right)$ and assume further that $A$ is a nonsingular curve with $g(A) \geqq 2$. Then the following assertions hold.

(1) If $\left(K_{V}, A\right) \geqq 0$ then $h^{0}(V, A) \leqq(1 / 2)\left(A^{2}\right)+2$. 
(2) If $\left(K_{V}, A\right) \leqq-1$ then $h^{0}(V, A) \leqq\left(A^{2}\right)+2-g(A)$.

Proof. (1) Suppose $\left(K_{V}, A\right) \geqq 0$. Then conditions of Lemma 2.6 with $X:=A$ and $L:=\left.A\right|_{A}$ are satisfied. Hence we have $c\left(\left.A\right|_{A}\right)=\left(A^{2}\right)+2\left(1-h^{0}\left(A, \mathcal{O}_{A}(A)\right)\right) \geqq 0$. This, together with the equality $h^{0}(V, A) \leqq 1+h^{0}\left(A, \mathcal{O}_{A}(A)\right)$, implies the assertion (1).

(2) Suppose $\left(K_{V}, A\right)<0$. Then $H^{1}\left(A, \mathcal{O}_{A}(A)\right) \cong H^{0}\left(A, \mathcal{O}_{A}\left(K_{V}\right)\right)=0$. Hence we have $h^{0}(V, A) \leqq 1+h^{0}\left(A, \mathcal{O}_{A}(A)\right)=\left(A^{2}\right)+2-g(A)$ by the Riemann-Roch theorem. The assertion (2) is proved.

Now we can prove the following:

Theorem 2.8. Assume that $A$ satisfies the condition (*) given at the beginning of $\$ 2$ and assume further that $V$ is not a rational surface. Then the following assertions hold true, where $A$ is replaced by a general member of $|A|$ is necessary.

(1) Suppose $h^{0}(V, A) \geqq\left(A^{2}\right)+1$. Then $h^{0}(V, A)=\left(A^{2}\right)+1=3, g(A)=p_{a}(A) \geqq 2$ and $1+p_{g}(V) \leqq h^{1}(V, A)+\chi\left(\mathcal{O}_{V}\right)=h^{0}\left(V, K_{V}-A\right)+g(A)$. Moreover, $\Phi_{|A|}: V \rightarrow \boldsymbol{P}^{2}$ is a morphism of degree two.

(2) Suppose $h^{0}(V, A)=\left(A^{2}\right)$. Then one of the following cases takes place.

(2-1) $V$ is an elliptic ruled surface with general members of $|A|$ as crosssections of the $\boldsymbol{P}^{1}$-fibration. $\Phi_{|A|}$ is a morphism of degree $\leqq 3$ onto a surface. (See the precise description of $V$ and $A$ in Proposition 2.5).

(2-2) We have $g(A)=p_{a}(A)=2$ and $\left(K_{V}, A\right) \leqq-1 . \quad V$ is a ruled surface satisfying $q(V)=h^{1}(V, A) \leqq 2 . \quad \Phi_{|A|}$ is a morphism of degree $\leqq 3$ onto a surface.

(2-3) We have $g(A)=p_{a}(A) \geqq 2,1+p_{g}(W) \leqq h^{1}\left(W, f^{\prime}(A)\right)+\chi\left(\mathcal{O}_{W}\right)=h^{0}\left(W, K_{W}-\right.$ $\left.f^{\prime}(A)\right)+g(A)-1$ and $\left(A^{2}\right)=3$ or 4 . Here $f:=i d$ if $B s|A|=\phi$ and $f: W \rightarrow V$ is the blowing-up of $a$ base point $P$ of $|A|$ if $B s|A| \neq \phi$. If $\left(A^{2}\right)=3$ and $|A|$ is base point free, then $g(A) \geqq 3$ and $\Phi_{|11|}: V \rightarrow \boldsymbol{P}^{2}$ is a morphism of degree three. If $\left(A^{2}\right)=3$ and $|A|$ has base points, then $|A|$ contains only one base point $P$ and $\Phi_{\left|f^{\prime}(A)\right|}: W \rightarrow \boldsymbol{P}^{2}$ is a morphism of degree two. If $\left(A^{2}\right)=4$, then $g(A) \geqq 3$ and $\Phi_{|A|}: V \rightarrow \boldsymbol{P}^{3}$ is a morphism of degree one (resp. or two) onto a quartic surface (resp. or a quardratic surface).

(3) Suppose $V$ is not a ruled surface. Then $h^{0}(V, A) \leqq(1 / 2)\left(A^{2}\right)+2$. Suppose further $h^{0}(V, A)=(1 / 2)\left(A^{2}\right)+2$. Then $g(A)=p_{a}(A) \geqq(1 / 2)\left(A^{2}\right)+1,1+p_{g}(V) \leqq h^{1}(V$, $A)+\chi\left(\mathcal{O}_{V}\right)=h^{0}\left(V, K_{V}-A\right)+g(A)+1-(1 / 2)\left(A^{2}\right)$, and either $\Phi_{|A|}: V \rightarrow \boldsymbol{P}^{N}(N:=$ $\left.h^{0}(V, A)-1\right)$ is a birational morphism onto a surface of degree $2(N-1)$ or $\Phi_{|A|}$ : $V \rightarrow \boldsymbol{P}^{N}$ is a morphism of degree two onto a normal rational surface $\bar{W}$ of degree $N-1$ in $\boldsymbol{P}^{N}$. (See Lemma 2.2 for the precise description of $\bar{W}$. )

Proof. (1) Suppose $h^{0}(V, A) \geqq\left(A^{2}\right)+1$. Since $V$ is not a rational surface, we have then $h^{0}(V, A)=\left(A^{2}\right)+1$ by Lemmas 2.1 and 2.2. By Lemma 2.4, $|A|$ is base point free. Hence $A$ is a nonsingular curve and $g(A)=p_{a}(A)$. Since $V$ 
is not a rational surface $A$ is not a rational curve, for otherwise $\Phi_{\left|h^{\prime}(A)\right|}: V^{\prime} \rightarrow$ $\boldsymbol{P}^{1}$ is a $\boldsymbol{P}^{1}$-fibration in the notation of Lemma 2.1. Therefore, $g(A) \geqq 2$ by Proposition 2.5. Then we have $\left(K_{V}, A\right) \geqq 0$ and $\left(A^{2}\right)+1=h^{0}(V, A) \leqq 1+h^{\circ}(A$, $\left.\mathcal{O}_{A}(A)\right) \leqq(1 / 2)\left(A^{2}\right)+2$ in view of Lemma 2.7. Hence we must have $h^{\circ}(V, A)=$ $\left(A^{2}\right)+1=3$ and $h^{0}(V, A)=1+h^{0}\left(A, \mathcal{O}_{A}(A)\right)$. Considering cohomologies of the following exact sequence

$$
0 \longrightarrow \mathcal{O}_{V} \longrightarrow \mathcal{O}_{V}(A) \longrightarrow \mathcal{O}_{A}(A) \longrightarrow 0,
$$

we obtain $1+p_{g}(V) \leqq h^{1}(V, A)+\chi\left(\mathcal{O}_{V}\right)=h^{0}\left(V, K_{V}-A\right)+g(A)$. The last assertion can be proved as in Lemma 2.1.

(2) Assume $h^{0}(V, A)=\left(A^{2}\right)$. We use the same arguments and notations $f: W \rightarrow V$ and $\sum_{i=1}^{b} m_{i}^{2}$ as in Lemma 2.1. Then $f^{\prime}(A)$ is a nonsingular curve and the geometric genus $g(A)=p_{a}\left(f^{\prime}(A)\right)$. Since $V$ is not a rational surface, we have $g(A) \geqq 1$.

Suppose $g(A)=1$. Then we have $\left(A^{2}\right)=h^{0}(V, A)=h^{0}\left(W, f^{\prime}(A)\right)=\left(f^{\prime}(A)\right)^{2}=$ $\left(A^{2}\right)-\sum_{i=1}^{b} m_{\imath}^{2}$ by Proposition 2.5. Hence we must have $\sum_{i=1}^{b} m_{i}^{2}=0, V=W$ and $p_{a}(A)=1$. So, the hypothesis of Proposition 2.5 is satisfied and hence the case $(2-1)$ occurs.

Suppose $g(A) \geqq 2$ and $\left(K_{W}, f^{\prime}(A)\right) \leqq-1$. Then $\kappa(V)=-\infty$ and $V$ is a ruled surface with $q(V) \leqq g(A)$ because $f^{\prime}(A)$ is nef. By Lemma 2.7, we have $\left(A^{2}\right)=$ $h^{0}\left(W, f^{\prime}(A)\right) \leqq 1+h^{0}\left(f^{\prime}(A), \mathcal{O}_{f^{\prime}(A)}\left(f^{\prime}(A)\right)\right)=\left(f^{\prime}(A)\right)^{2}+2-g(A)$. Hence we must have $g(A)=2,\left(A^{2}\right)=\left(f^{\prime}(A)\right)^{2}$, i. e., $V=W$ and $|A|$ is base point free, and $h^{0}(V, A)$ $=1+h^{\circ}\left(A, \Theta_{A}(A)\right)$. By considering the same exact sequence as in (1), we can prove $h^{1}(V, A)=q(V)$. This fits the case (2-2). Indeed, the last assertion in the case (2-2) can be proved similarly as in Lemma 2.1.

Suppose $g(A) \geqq 2$ and $\left(K_{W}, f^{\prime}(A)\right) \geqq 0$. We shall show that the case (2-3) occurs. In view of Lemma 2.7 , we have $\left(A^{2}\right)=h^{0}\left(W, f^{\prime}(A)\right) \leqq 1+h^{0}\left(f^{\prime}(A)\right.$, $\left.\mathcal{O}_{f^{\prime}(A)}\left(f^{\prime}(A)\right)\right) \leqq(1 / 2)\left(A^{2}\right)+2-(1 / 2) \sum_{i=1}^{b} m_{i}^{2}$. So we must have $h^{0}(V, A)=\left(A^{2}\right)=3$ or 4 , and $h^{0}\left(W, f^{\prime}(A)\right)=1+h^{0}\left(f^{\prime}(A), \mathcal{O}_{f^{\prime}(A)}\left(f^{\prime}(A)\right)\right)$. Moreover, if $\left(A^{2}\right)=4$, then $\sum_{i=1}^{b} m_{i}^{2}=0$, i. e., $V=W$ and $|A|$ is base point free, and if $\left(A^{2}\right)=3$, then $\sum_{i=1}^{b} m_{\imath}^{2}$ $\leqq 1$. Then $g(A)=p_{a}(A)$ and the last three assertions of (2-3) follow (cf. Lemmas 2.1 and 2.4). In fact, $g(A)=1+(1 / 2)\left(f^{\prime}(A), K_{W}+f^{\prime}(A)\right) \geqq 1+(1 / 2)\left(f^{\prime}(A)\right)^{2}$. Considering an exact sequence similar to the one given in (1) with $V$ (resp. $A$ ) replaced by $W$ (resp. $f^{\prime}(A)$ ), we can prove that $1+p_{g}(W) \leqq h^{1}\left(W, f^{\prime}(A)\right)+\chi\left(\mathcal{O}_{W}\right)$ $=h^{0}\left(W, K_{W}-f^{\prime}(A)\right)+g(A)-1$. So all assertions in the case (2-3) are verified.

(3) Suppose $V$ is not a ruled surface. We use the notation $f: W \rightarrow V$ in Lemma 2.1. By the additional assumption, we have $g(A)=p_{a}\left(f^{\prime}(A)\right) \geqq 2$ (cf. Proposition 2.5) and $\left(K_{W}, f^{\prime}(A)\right) \geqq 0$ because $f^{\prime}(A)$ is nef. Then we have $h^{\circ}(V$, $A)=h^{0}\left(W, f^{\prime}(A)\right) \leqq 1+h^{0}\left(f^{\prime}(A), \mathcal{O}_{f^{\prime}(A)}\left(f^{\prime}(A)\right)\right) \leqq(1 / 2)\left(f^{\prime}(A)\right)^{2}+2 \leqq(1 / 2)\left(A^{2}\right)+2$ in view of Lemma 2.7 .

Consider the case $h^{0}(V, A)=(1 / 2)\left(A^{2}\right)+2$. Then $\left(f^{\prime}(A)\right)^{2}=\left(A^{2}\right)$, i.e., $V=W$ and $|A|$ is base point free, and $h^{\circ}(V, A)=1+h^{0}\left(A, \mathcal{O}_{A}(A)\right)$. Note that $\operatorname{deg} \Phi_{\mid A}$ : 
$\equiv\left(A^{2}\right) /(1 / 2)\left(A^{2}\right)$ as in Lemma 2.1. Then the last assertion follows. Note that $g(A)=1+(1 / 2)\left(A, K_{V}+A\right) \geqq(1 / 2)\left(A^{2}\right)+1$ because $A$ is nef. By considering the same exact sequence as in (1), we obtain $1+p_{g}(V) \leqq h^{1}(V, A)+\chi\left(\mathcal{O}_{V}\right)=h^{0}\left(V, K_{V}\right.$ $-A)+g(A)+1-(1 / 2)\left(A^{2}\right)$. The assertion (3) is proved.

In order to prove Theorem 2.10, we need the following preparation. Write $\left|K_{V}+D\right|=|C|+G$ as in the statement of Theorem 2.10. Define $\boldsymbol{Q}$-divisors $C^{*}$ and $G^{*}$ in the same fashion as for $F^{*}$ and $G^{*}$ before Lemma 1.1. Then we can also show that $K_{V}+D^{*} \equiv C^{*}+G^{*}, C^{*} \geqq C$ and $0 \leqq G^{*} \leqq G$ as in Lemma 2.1. Moreover, we have:

Lemma 2.9. (1) We have $\left(K_{V}+D^{*}\right)^{2} \geqq\left(C^{2}\right)$.

(2) If (1) becomes an equality, then $D^{*}$ is an integral divisor and $C \sim K_{V}+D^{*}$.

Proof. (1) Since $K_{V}+D^{*}, C^{*}$ and $C$ are nef, we havc $\left(K_{V}+D^{*}\right)^{2}=\left(K_{V}+\right.$ $\left.D^{*}, C^{*}+G^{*}\right) \geqq\left(K_{V}+D^{*}, C^{*}\right)=\left(C^{*}+G^{*}, C^{*}\right) \geqq\left(C^{*}\right)^{2}=\left(C, C^{*}\right) \geqq\left(C^{2}\right)$.

(2) Suppose that (1) is an equality. Then by the proof of (1), we have $\left(K_{V}+D^{*}, G^{*}\right)=0$, and $\left(C^{*}-C\right)^{2}=\left(C^{*}\right)^{2}-2\left(C, C^{*}\right)+\left(C^{2}\right)=0$. Hence $C^{*}=C$ because $\operatorname{Supp}\left(C^{*}-C\right) \subseteq \operatorname{Supp}\left(D-D^{*}\right)$ which has negative definite intersection matrix. Note that $\left(C, G^{*}\right)=\left(C, K_{V}+D^{*}-C\right)=\left(C+G^{*}, K_{V}+D^{*}\right)-\left(C^{2}\right)=\left(K_{V}+D^{*}\right)^{2}-\left(C^{2}\right)=0$ and $\left(G^{*}\right)^{2}=\left(K_{V}+D^{*}-C, G^{*}\right)=0$. Hence, $G^{*} \equiv 0$ by the Hodge index theorem. Indeed, $G^{*}=0$ because $G^{*} \geqq 0$. Hence $\operatorname{Supp} G \subseteq \operatorname{Supp}\left(D-D^{*}\right)$ and $G-\left(D-D^{*}\right) \equiv$ $K_{V}+D-C-\left(D-D^{*}\right) \equiv K_{V}+D^{*}-C^{*}-G^{*} \equiv 0 . \quad$ Since $\operatorname{Supp}\left(G+D^{*}-D\right)(\subseteq \operatorname{Supp}(D-$ $\left.D^{*}\right)$ ) has negative definite intersection matrix, we have $G=D-D^{*}$. So, $D^{*}$ is an integral divisor and $C \sim K_{r^{r}}+D-G=K_{V}+D^{*}$.

Now Theorem 2.10 which is stated in the Introduction of the article in a consequence of Lemmas 2.1, 2.2 and 2.9 and Theorem 2.8. In fact, note that $p_{a}(C)=1+(1 / 2)\left(C, K_{V}+C\right) \leqq 1+(1 / 2)\left(C^{2}\right)+(1 / 2)\left(C, K_{V}+D^{*}\right)$.

Sakai [6; Theorem 6.5] proved the inequality $\bar{p}_{g} \leqq(1 / 2)\left(K_{V}+D^{*}\right)^{2}+2$ in Theorem 2.10 , (3), provided that $D$ is semi-stable.

In the forthcoming article [7], we shall give first the geometric structure of those pairs $(V, D)$ satisfying $\bar{p}_{g}=(1 / 2)\left(\bar{c}_{1}^{2}\right)+2$, where $\left(\bar{c}_{1}^{2}\right):=\left(K_{V}+D^{*}\right)^{2}$. Then we shall verify an inequality of the form $\left(\bar{c}_{1}^{2}\right) \geqq \alpha \bar{c}_{2}+\beta$, where $\bar{c}_{2}:=c_{2}(V)-e(D)$ is the Euler number of $V-D$, and $\alpha(>0)$ and $\beta$ are two rational numbers. Finally, we shall give the geometric structure of those pairs $(V, D)$ satisfying $\left(\bar{c}_{1}^{2}\right)=\alpha \bar{c}_{2}+\beta$.

\section{References}

[1] Fujita, T., On the structure of polarized varieties with $\Delta$-genera zero, J. Fac. Sci. Univ. Tokyo Sec. IA Math., 22 (1975), 103-115.

[2] Hartshorne, R., Algebraic Geomctry, New York, Heidelberg, Berlin, Springer- 
Verlag, 1977.

[. 3 ] Martens, H.H., Varieties of special divisors on a curve. II, J. reine angew. Math., 233 (1969), 89-100.

[4] Miyanishi, M. and Tsunoda, S., Open algebraic surfaces with Kodaira dimension $-\infty$, Proceedings of Symposia in Pure Mathematics, 46 (1987), 435-450.

[5] Nagata, M., On rational surfaces I, Memoirs of the College of Science, University of Kyoto, Series A, 32 (1960), 351-370.

[6] Sakai, F., Semi-stable curves on algebraic surfaces and logarithmic pluricanonical maps, Math. Ann., 254 (1980), 89-120.

[7] Zhang, D.-Q., Noether's inequality for non-complete algebraic surfaces of general type, II, forthcoming. 